\title{
Long Persistent Luminescence Enabled by Dissociation of Triplet Intermediate States in an Organic Guest/Host System
}

Leixin Xiao, ${ }^{\dagger, \S}$ Zhiwei Wang, ${ }^{\dagger, \S}$ Chunfeng Zhang, ${ }^{\dagger,}$ Xiaoyu Xie, ${ }^{\ddagger}$ Haibo Ma, ${ }^{\ddagger}$

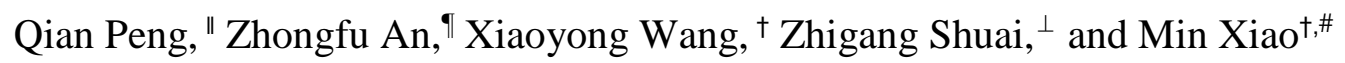

${ }^{\dagger}$ National Laboratory of Solid State Microstructures, School of Physics, and Collaborative Innovation Center for Advanced Microstructures, Nanjing University, Nanjing 210093, China

‡ School of Chemistry and Chemical Engineering, Nanjing University, Nanjing 210093, China, Nanjing University, Nanjing 210093, China

" China Key Laboratory of Organic Solids and Beijing National Laboratory for Molecular Science, Institute of Chemistry, Chinese Academy of Sciences, Beijing 100190, China

" Key Laboratory of Flexible Electronics and Institute of Advanced Materials, Nanjing Tech University, Nanjing 211816, China

${ }^{\perp}$ Department of Chemistry and MOE Key Laboratory of Organic Optoelectronics and Molecular Engineering, Tsinghua University, Beijing 10084, China

\# Department of Physics, University of Arkansas, Fayetteville, Arkansas 72701, United States 
Materials and Methods

Figure S1. Charge transfer dynamics in a blend TMB/PPT film.

Figure S2. Dependence of carrier recombination dynamics on excitation density.

Figure S3. Global fitting analysis of ns-resolved TA data of a TMB/PPT blend.

Figure S4. Natural transition orbital analysis for low-lying excited states.

Figure S5. Calculated energy alignment of excited states with different configurations.

Figure S6. Comparison of TA spectra of intermediate triplets in a TMB/PPT blend film and ${ }^{3} \mathrm{LE}$ of TMB.

Figure S7. Spectroelectrochemitry measurements of spectral features of the $\mathrm{TMB}^{+}$ and $\mathrm{PPT}^{-}$.

Figure S8. Temperature-dependent dynamics of charge separation and carrier diffusion in a TMB/PPT blend film.

Figure S9. Temperature-dependent PL emission and decay dynamics of TMB/PPT and reference films.

Figure S10. Nomarlized spectra for references.

Table S1. Energy alignment of low-lying excited states with different configurations. 


\section{Materials and Methods}

\section{Sample preparation}

The TMB and PPT powders were purchased from Tokyo Chemical Industry Co., Ltd. (Shanghai) and Luminescence Technology Corp. (Taiwan). We used a conventional melting-cast method to fabricate films of TMB, PPT and TMB/PPT blends. For the optical measurements of TMB/PPT blend film, we placed a mixture of TMB and PPT powders on a quartz plate and heated it up to $250{ }^{\circ} \mathrm{C}$ for 10 seconds. The plate was cooled rapidly to room temperature and mounted on a cold finger of a cryostat (Microstat He, Oxford) where vacuum was established. All operations were processed in a glovebox filled with nitrogen gas.

The reference sample of TMB-doped ZOENOR polymer was prepared as a reference sample to study the dynamics of the ${ }^{3} \mathrm{LE}$ state at TMB. The cyclic olefin copolymer ZEONOR provide a nonpolar and rigid amorphous environmental without forming a CT state with TMB ${ }^{1}$. The ZEONOR 1060R was purchased from Zeon Chemicals (Shanghai). We used a drop-cast method to fabricate films of TMB/ZEONOR.

\section{Photoluminescence measurements}

PL spectra were recorded using a liquid-nitrogen cooled Silicon charge-coupled device (CCD) mounted on a monochromator. A 320-nm beam generated by an optical parametric amplifier (OperA Solo, Coherent Inc.) pumped by a Ti:sapphire regenerative amplifier (Libra, Coherent Inc.) was used to excite PL from the samples. LPL spectra of TMB/PPT blends were recorded with a 340-nm LED that was triggered by a synthesized signal generator (TFG3605, Suin) with a repetition rate of $1.67 \mathrm{mHz}$ and duty ratio of $10 \%$. The emission spectra at different delays were recorded with an exposure time of $100 \mathrm{~ms}$. TRFL spectra were recorded by the technique of timecorrelated single-photon counting (Picoquant, Picoharp 300) using an avalanche photodiode having a temporal resolution of 50 ps (Picoquant, MPD).

\section{Transient absorption spectroscopy.}

TA spectroscopic measurements were performed using a home-built system with a Ti:sapphire regenerative amplifier (Libra, Coherent Inc.) and a synchronized frequency-tripled sub-nanosecond laser (Picolo AOT MOPA, InnoLas) at $355 \mathrm{~nm}$ 
(pulse duration $\sim 0.8 \mathrm{~ns})^{2-3}$. The probe beam was a broadband chirp-corrected supercontinuum light source generated by focusing a small portion of the femtosecond laser beam onto either a $3 \mathrm{~mm}$-thick sapphire plate for the visible range or a $4 \mathrm{~mm}$-thick YAG plate for the infrared range. The differential transmission in visible and infrared range was analyzed by a silicon CCD (S11071, Hamamatsu) and a GaAs camera (G11608, Hamamatsu), respectively, mounted on a monochromator (Acton 2358, Princeton Instrument) at $1 \mathrm{kHz}$ enabled by a custom-built control board. For fs-resolved TA measurements, an optical parametric amplifier (OperA Solo, Coherent Inc.) pumped by the regenerative amplifier was used to generate the pump beam at the different wavelengths. For ns-resolved TA spectroscopy, the pump beam was replaced by the $355 \mathrm{~nm}$ sub-nanosecond laser. The laser was synchronized to the probe pulse with a desired delay by an electronic delay generator (SRS DG645, Stanford Research System). For $\mu$ s-resolved TA measurements, the pump beam of the sub-nanosecond 355-nm laser was set at a repetition frequency at $2 \mathrm{~Hz}$. A quartz halogen lamp (SLS301, Thorlabs) was used as the probe light source. A laser shutter (SR475, Stanford Research System) at $1 \mathrm{~Hz}$ with a duty cycle of 50\%, synchronized with the pump laser, was used to modulate the probe light. The transmission change was then analyzed by synchronized detectors with exposure time of $100 \mu$ s at different time delays.

\section{ESR measurements}

Solid state X-band ( 9.4 GHz) ESR spectra were recorded as the first derivative of absorption using a Bruker E500 spectrometer. All samples were prepared using the melt-casting method described above and then transferred to quartz tubes in the glovebox. The ESR spectra of TMB, PPT and their blend were recorded with and without photoirradiation by a 340-nm LED at $77 \mathrm{~K}$.

\section{Quantum chemical calculation}

To investigate the relative energy order of low-lying excited states (for both singlet and triplet states) in the blend system, we performed quantum chemical (QM) calculation for a TMB/PPT dimer system with initial geometries sampled from the trajectories of molecular dynamics (MD) simulation. 
The MD simulation was performed with the NAMD package suite ${ }^{4}$. The force fields of two molecules (TMB and PPT) generated by LigParGen and packmol package ${ }^{5-8}$ were used to obtain amorphous structures of a blend system with 50 TMB molecules and 450 PPT molecules. Two initial structures were constructed to generate two MD trajectories. After the NVT simulations at $300 \mathrm{~K}$ (total time was 100 ps with 1.0 fs per step), we assumed that the system was relaxed and the NPT simulations were performed at $1 \mathrm{~atm}$ pressure and $300 \mathrm{~K}$ (total time was $10 \mathrm{~ns}$ with $1.0 \mathrm{fs}$ per step). Langevin dynamics and Nose-Hoover-Langevin algorithm were used to maintain the temperature and the pressure of the system respectively. From the NPT trajectories in the time range of 5-10 ns, twenty TMB/PPT pairs with the distance between the mass centers of TMB and PPT smaller than $6.5 \AA$ were selected randomly.

For the QM part, density functional theory (DFT) and time-dependent DFT (TDDFT) methods were used with the Gaussian package (version D.01) ${ }^{9}$. The first ten singlet and ten triplet excited states were computed using the TDDFT method after the geometry optimization via the DFT method for all 20 dimers at the B3LYP/6-31g(d, p) calculation level. To analyze the excitation behavior of the low-lying excited states, natural transition orbital (NTO) ${ }^{10}$ analyses were performed for all dimers. According to the different behaviors of the energic orders and excitation characteristics, the 20 dimers can be classified into four groups with different intermolecular interaction strengths, as listed in Table S1. The low-lying excited states of dimers with larger interactions (namely type I and II, total number: ten) show small energy gaps between locally excited triplet states and CT state (Figure S4 displays the NTO analysis of lowlying excited states of the type I example in Table S1). In contrast, the excitation energies of ${ }^{1} \mathrm{CT} /{ }^{3} \mathrm{CT}$ states for type III (total number: five) and type IV (total number: four) are larger and degenerate.

A dimer model with a face-to-face $\pi-\pi$ stacking structure is built to investigate the relation between the energy order of low-lying excited states and intermolecular interaction. This model contains a series of dimers with similar $\pi-\pi$ stacking conformations but different intermolecular distances. We computed the low-lying singlet and triplet excited states of TMB and PPT molecules and all dimers in this model 
(Figure S5). The results are similar to the energy order listed in table S1. For the short distance $\mathrm{r}_{1}$, the energy gap between ${ }^{3} \mathrm{CT}$ and ${ }^{3} \mathrm{D}$ was small $(\sim 30 \mathrm{meV})$ and the gap between ${ }^{1} \mathrm{CT}$ and ${ }^{3} \mathrm{CT}$ implies the presence of intermolecular interactions. Conversely, ${ }^{1} \mathrm{CT}$ and ${ }^{3} \mathrm{CT}$ are almost degenerate for the dimer with a large distance $\mathrm{r}$. 

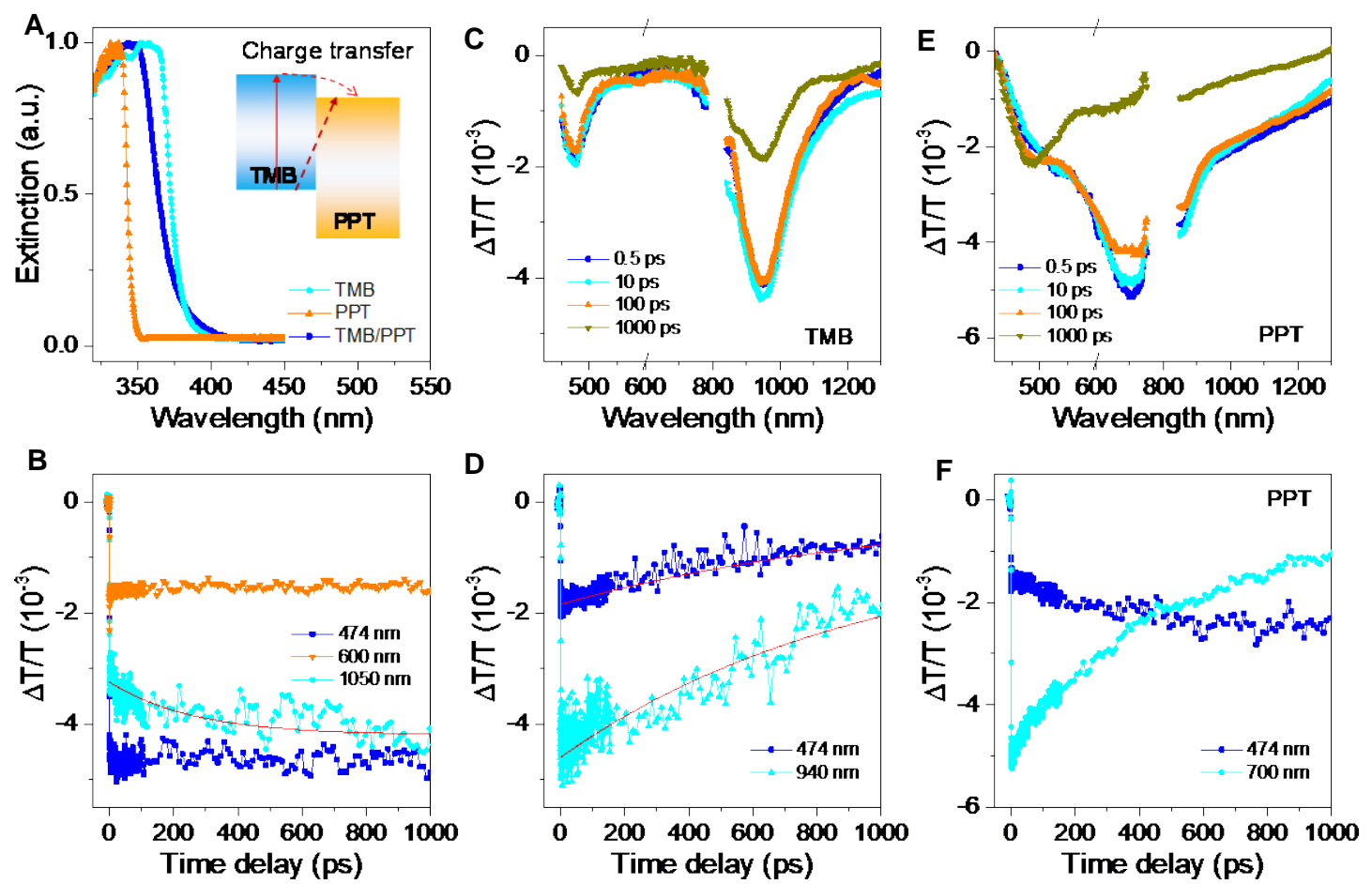

Figure S1. Charge transfer dynamics in a blend TMB/PPT film. (A)Extinction spectra of a TMB/PPT blend film and neat TBM and PPT films. Inset shows the scenario of ${ }^{1} \mathrm{CT}$ formation in the TMB/PPT blend through direct optical excitation or interfacial charge transfer upon optical excitation at $360 \mathrm{~nm}$. Most local excitations at TMB funnel into the CT state immediately. (B) Kinetic traces probed at 474 and $1040 \mathrm{~nm}$ in the TMB/PPT blend. The experimental conditions were the same as in Fig. 2 in the main text with pump at $360 \mathrm{~nm}$. (C) TA spectra recorded at different delays from a neat TMB film with pump at $360 \mathrm{~nm}$. (D) Kinetic traces probed at $474 \mathrm{~nm}$ and $940 \mathrm{~nm}$ probed in the neat TMB film. (E) TA spectra recorded at different delays from a neat PPT film with pump at $340 \mathrm{~nm}$. (F) Kinetic traces probed at $474 \mathrm{~nm}$ and $700 \mathrm{~nm}$ probed for the neat TMB film. 


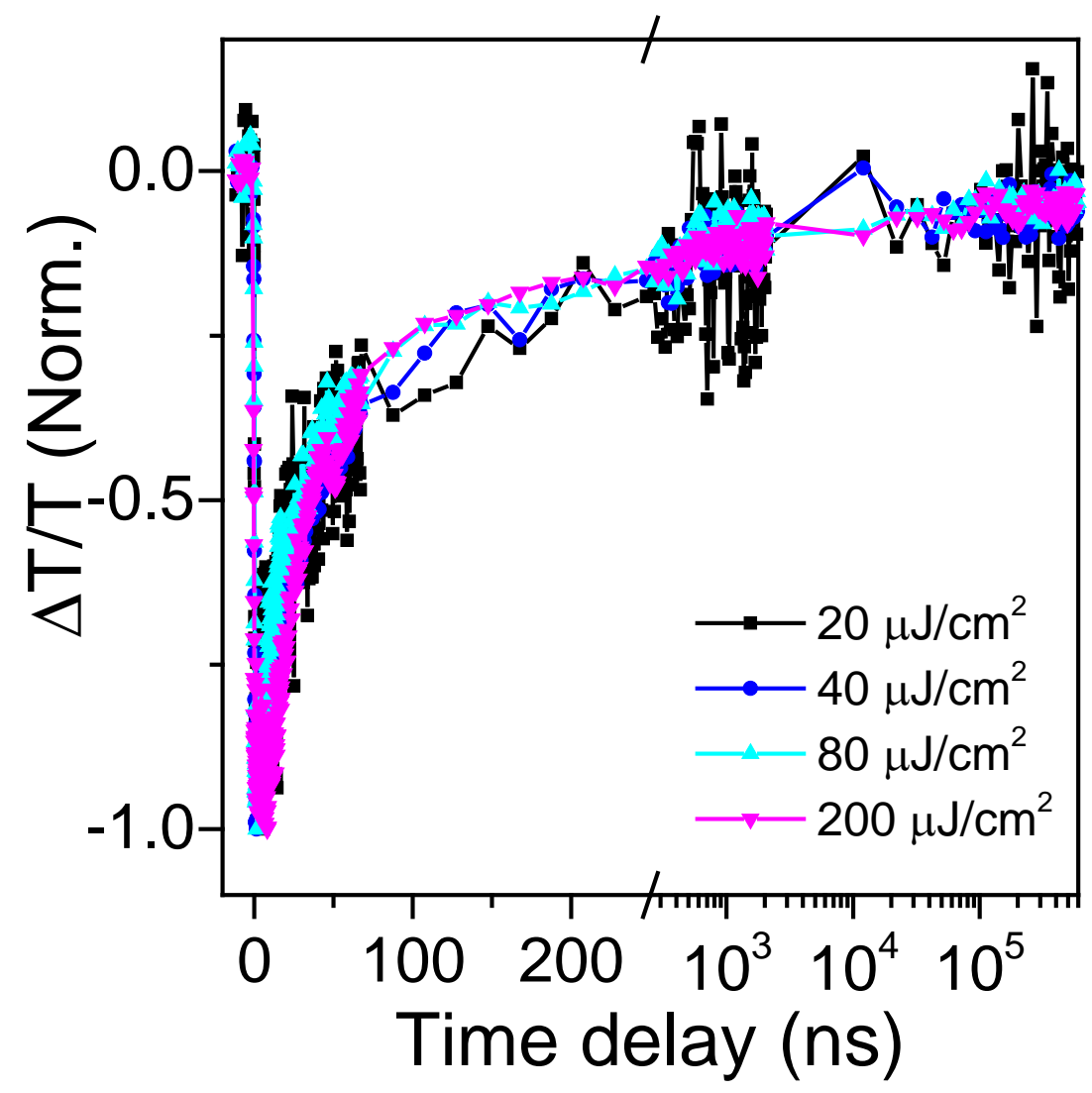

Figure S2. Dependence of carrier recombination dynamics on excitation density. Normalized kinetic curves probed at $1050 \mathrm{~nm}$ were recorded upon pump with different fluences. The wavelength of pump source is $355 \mathrm{~nm}$. 

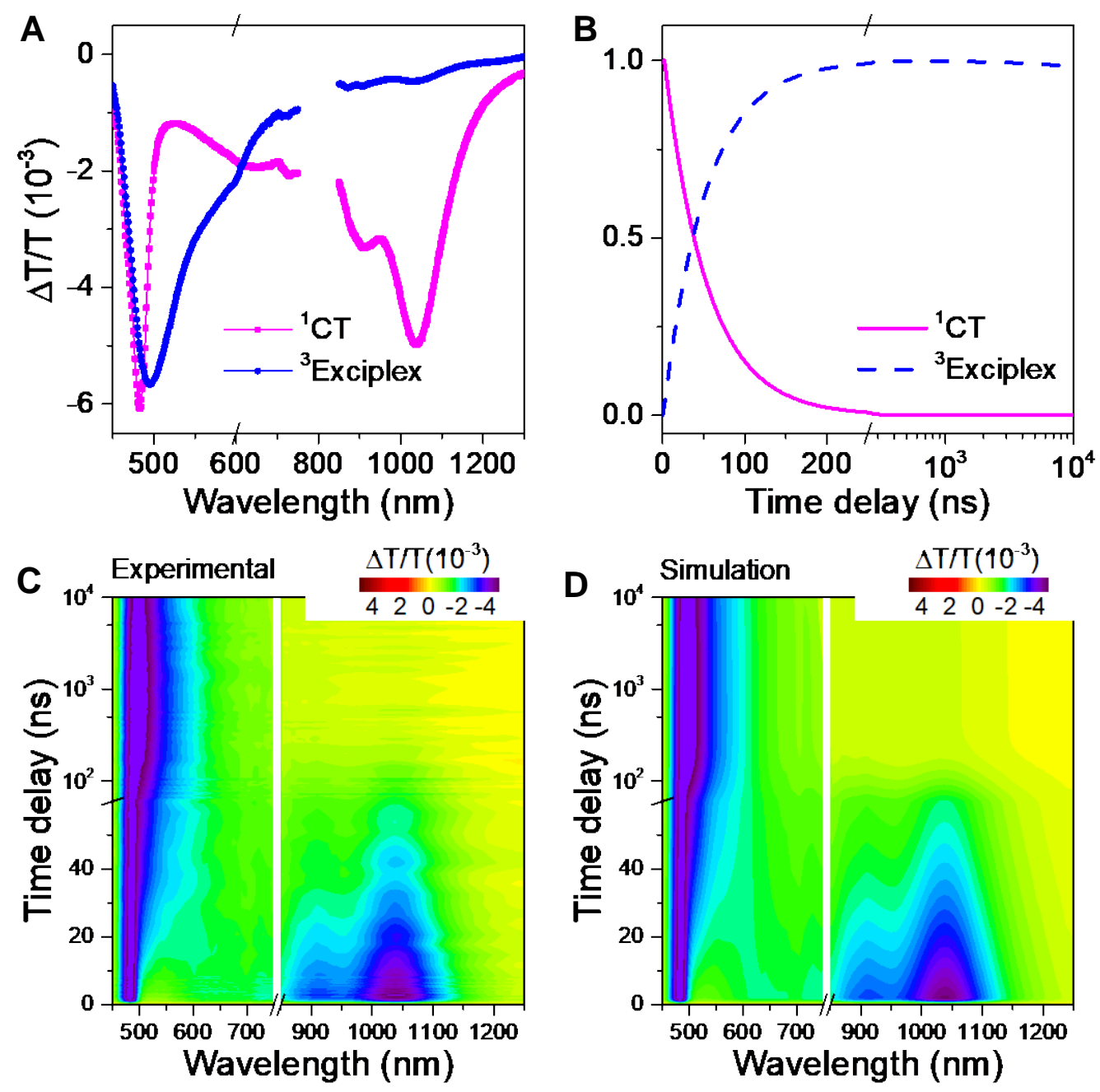

Figure S3. Global fitting analysis of ns-resolved TA data of a TMB/PPT blend. (A) Spectral characteristics and (B) temporal kinetics of the two components used to reproduce the TA data. The wavelength of pump source is $355 \mathrm{~nm}$. The two components can be assigned to exciplex ${ }^{1} \mathrm{CT}$ and triplet states. (C) Experimental and (D) simulation results of the TA data. 
A

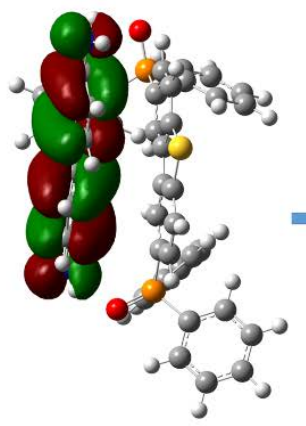

C
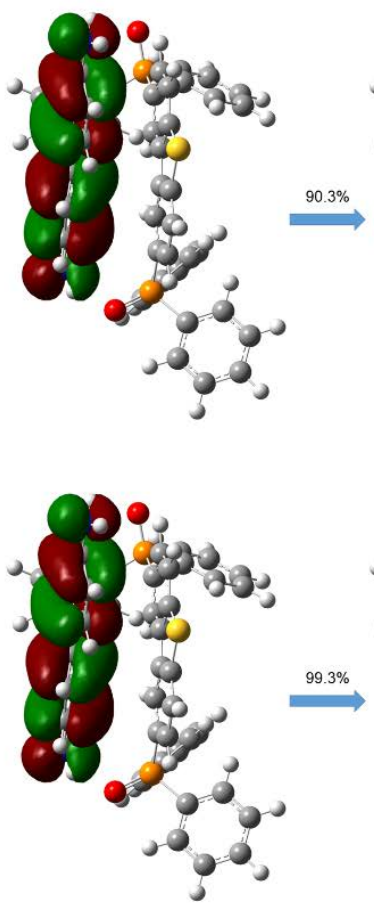

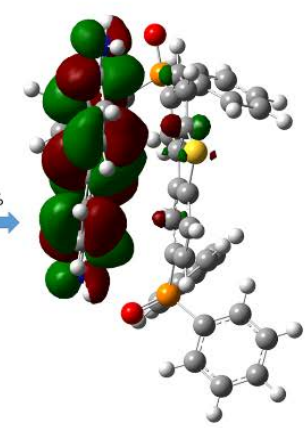

B
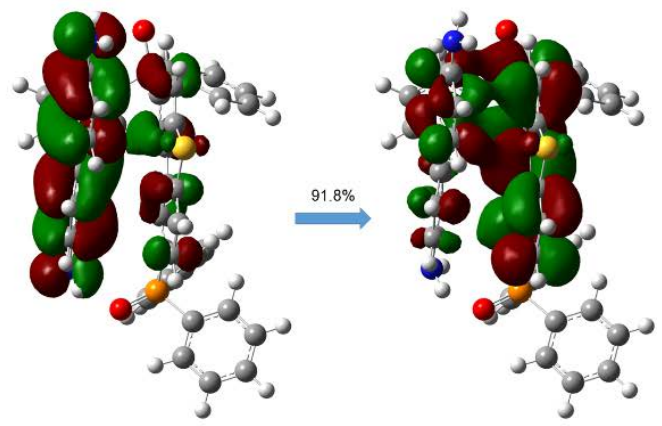

D
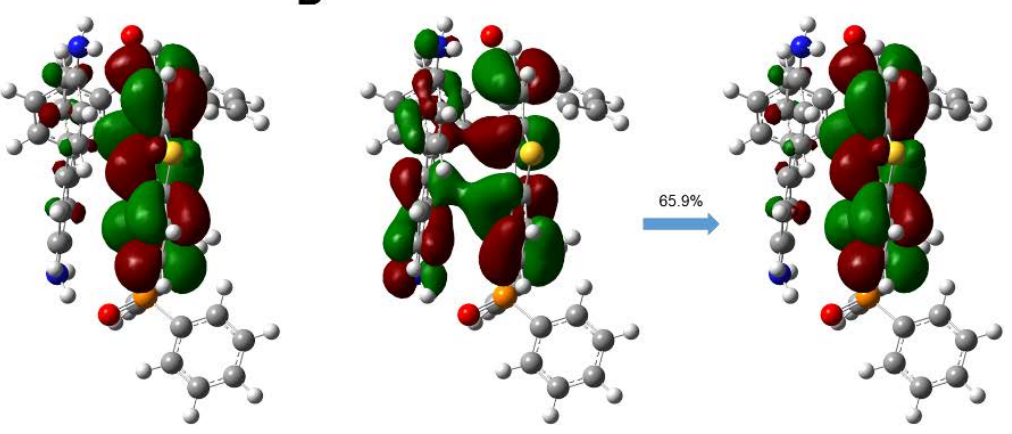

Figure S4. Natural transition orbital analysis for low-lying excited states. (A) $\mathrm{T}_{1}$ states with $\Delta E_{\mathrm{T} 1}=3.30 \mathrm{eV}$, (B) $\mathrm{T}_{2}$ states with $\Delta E_{\mathrm{T} 2}=3.42 \mathrm{eV}$, (C) $\mathrm{S}_{1}$ states with $\Delta E_{\mathrm{S} 1}=3.57$ $\mathrm{eV}$ and (D) $\mathrm{T}_{3}$ states with $\Delta E_{\mathrm{T} 3}=3.67 \mathrm{eV}$ of the type I dimer in Table S1 with optimized equilibrium geometry. The percentage indicated over the arrow means the weight of electron (left) -hole (right) excitation to the overall transition. 


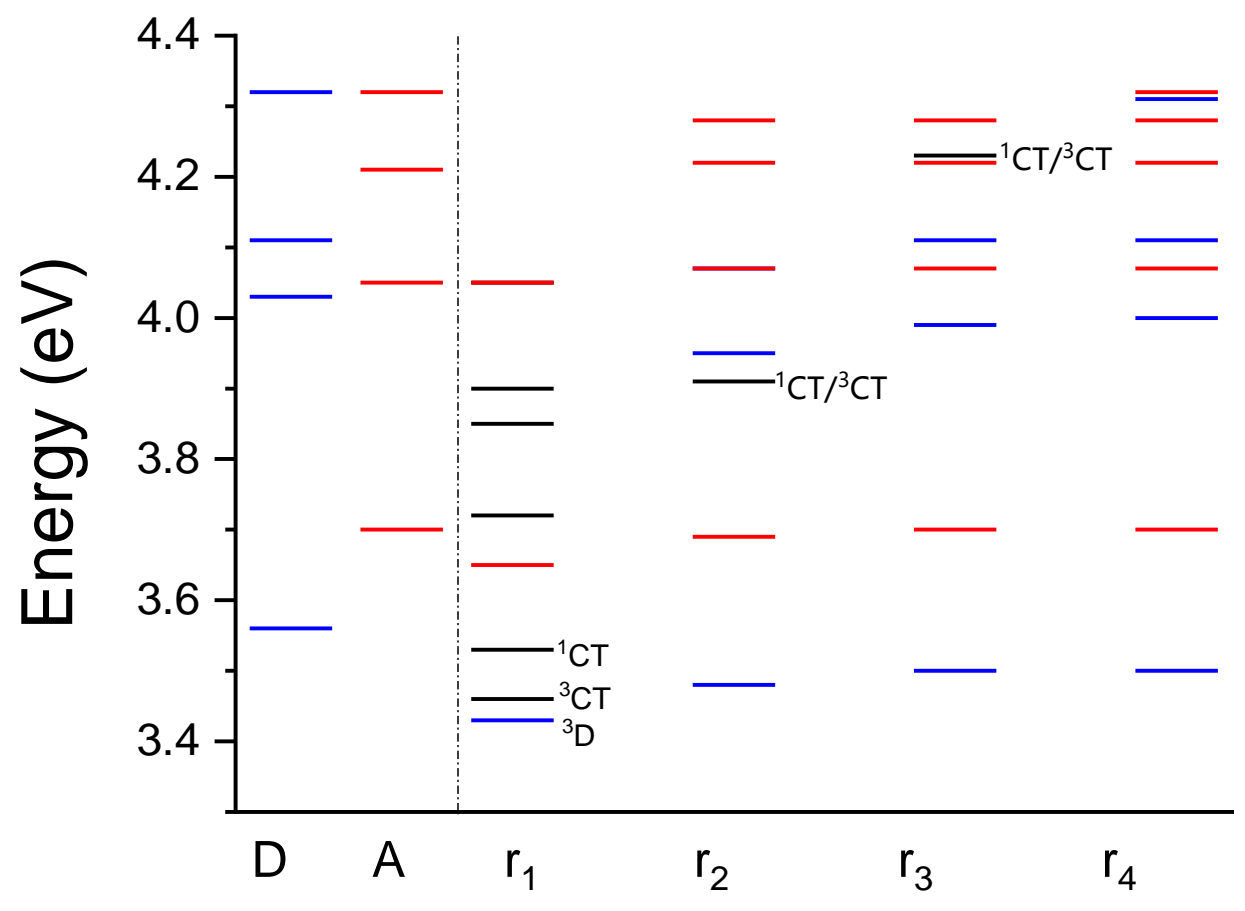

Figure S5. Calculated energy alignment of excited states with different configurations. Illustration of the energic levels of excited states (both singlet and triplet states) for model dimers and the triplet states of TMB (donor D, the blue lines) and PPT (acceptor $\mathrm{A}$, the red lines) in the energy window of $3.4-4.4 \mathrm{eV}$. For dimer systems, blue lines and red lines represent triplet states localized at a TMB molecule and PPT molecule respectively, while black lines show the energetic positions of states with CT or delocalized feature and the notation ${ }^{1 / 3}{ }^{1 /} \mathrm{CT}$ ' indicates the states of our target ${ }^{1} \mathrm{CT}$ and ${ }^{3} \mathrm{CT}$ states. Here the distances between TMB and PPT in dimers are around $\mathrm{r}_{1}=3.4 \AA$, $\mathrm{r}_{2}=5.3 \AA, \mathrm{r}_{3}=6.7 \AA$ and $\mathrm{r}_{4}=8.4 \AA$ individually. 


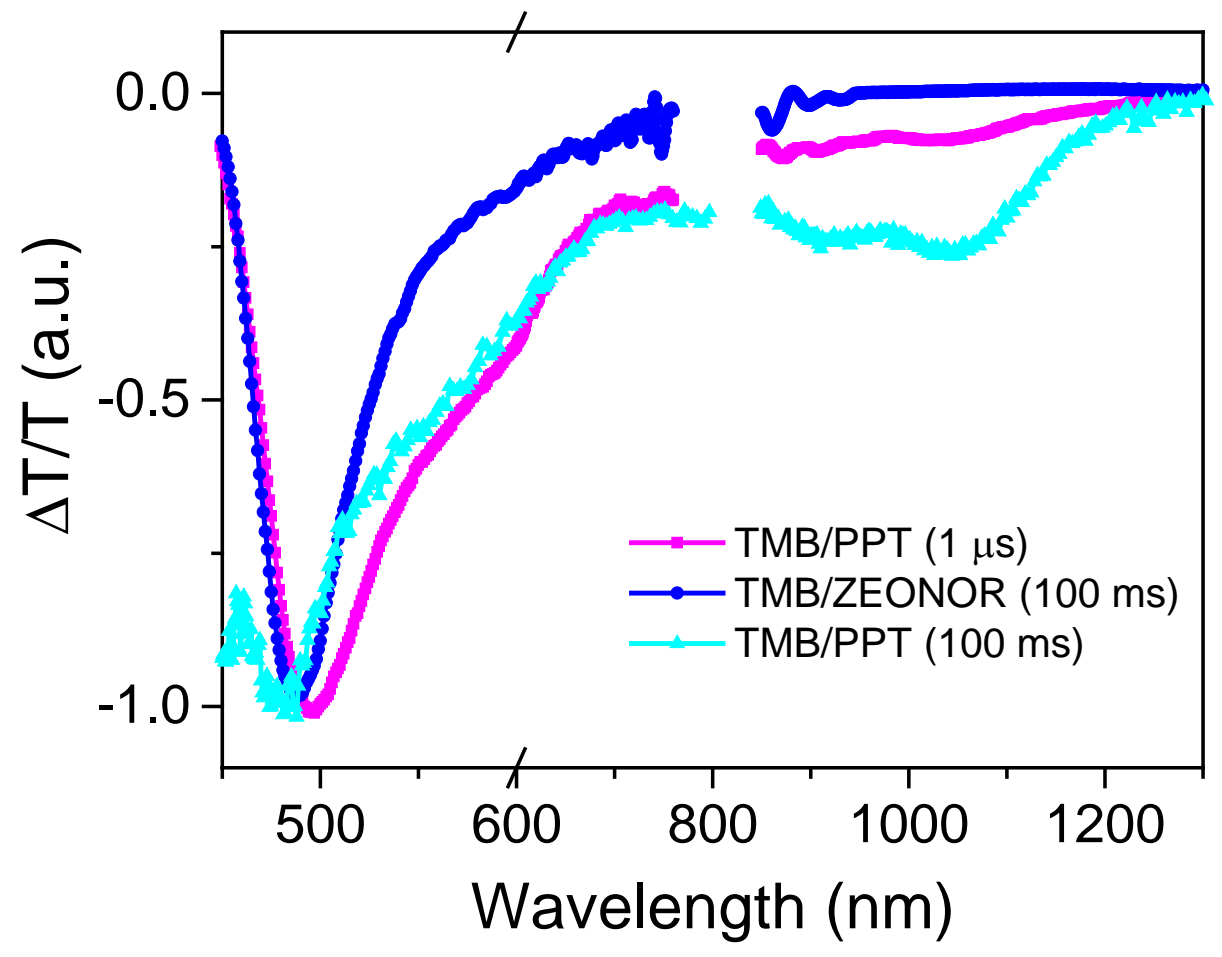

Figure S6. Comparison of TA spectra of intermediate triplets in a TMB/PPT blend film and ${ }^{3} \mathrm{LE}$ of TMB. Normalized TA spectra of the TMB/PPT blend recorded at the delays of $1 \mu \mathrm{s}$ and $100 \mathrm{~ms}$. A TA spectrum of the ${ }^{3} \mathrm{LE}$ state of TMB in a sample of TMB/ZEONOR is shown for comparison. The data were recorded at room temperature. In the TA spectra, the ESA feature of the triplet intermediate state appears at a wavelength range close to that of the ${ }^{3} \mathrm{LE}$ state. Nonetheless, the spectral feature of the triplet intermediates is much broader, implying that the triplet intermediate state has some similarity to but is not all the same characteristics as the ${ }^{3} \mathrm{LE}$ state, which is consistent with the triplet intermediates of mixed ${ }^{3} \mathrm{LE}$ and ${ }^{3} \mathrm{CT}$ states. The wavelength of pump source is $355 \mathrm{~nm}$. 

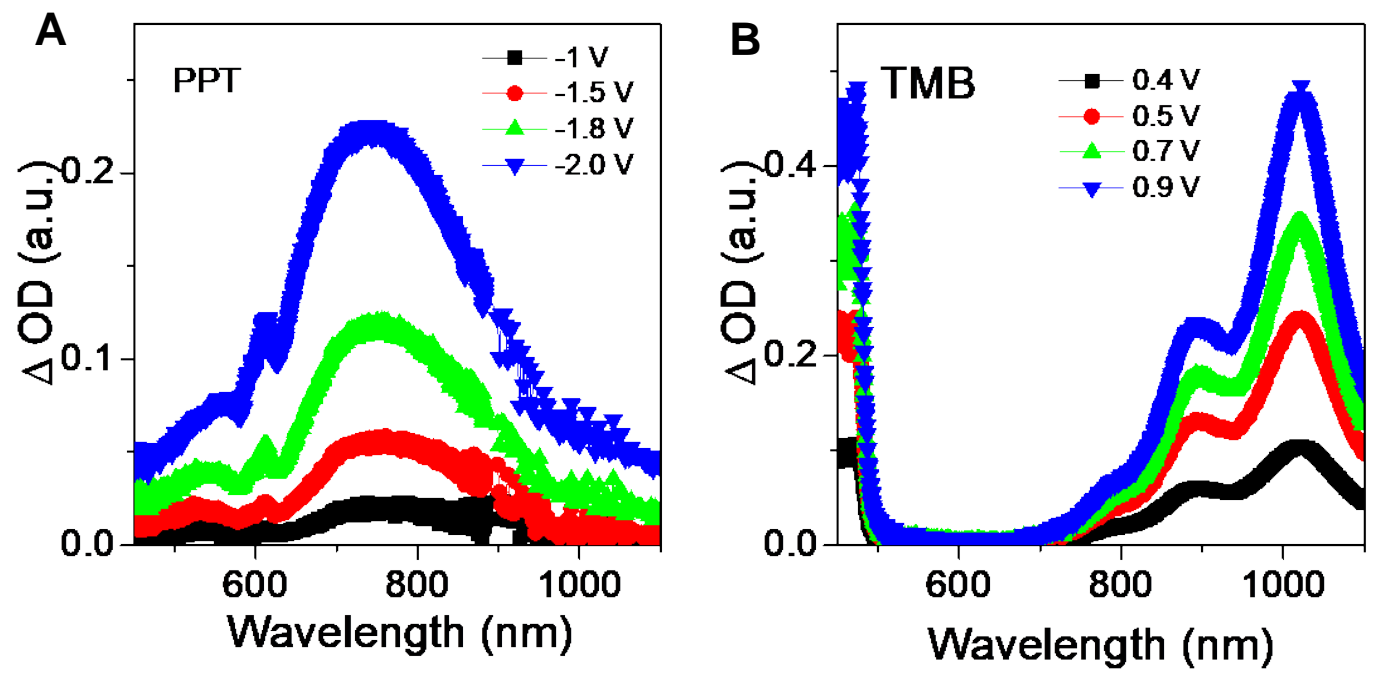

Figure S7. Spectroelectrochemitry measurements of spectral features of the $\mathrm{TMB}^{+}$and $\mathrm{PPT}^{-}$. Absorption spectra of (A) $\mathrm{PPT}^{-}$and (B) $\mathrm{TMB}^{+}$introduced by applying different voltages on the working electrode. Experiments were performed using a three-electrode system with a platinum mesh as the working electrode, a platinum wire as the counter electrode and $\mathrm{Ag} / \mathrm{AgCl}$ reference electrode. The measurements were performed with PPT and TMB dissolved in N,N-dimethylformamide and dichloromethane at a concentration of $0.005 \mathrm{~mol} / \mathrm{L}$, respectively, together with $0.1 \mathrm{~mol} / \mathrm{L}$ of tetrabutylammonium hexafluorophosphate as the supporting electrolyte. 

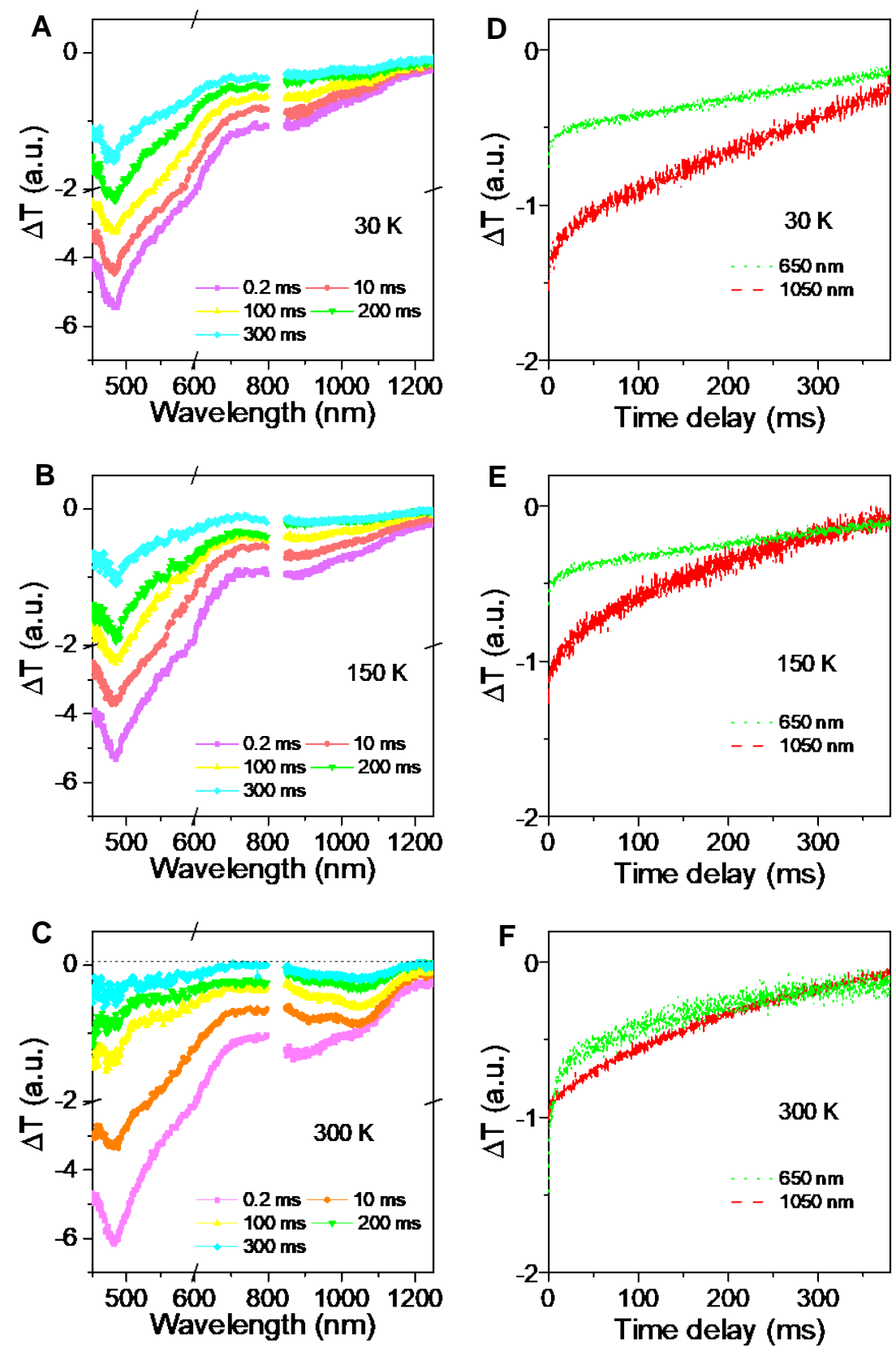

Figure S8. Temperature-dependent dynamics of charge separation and carrier diffusion in a TMB/PPT blend film. (A-C) Ms-resolved TA spectra in the TMB/PPT blend film at different time delays recorded at different temperatures. (D-F) Ms-resolved kinetics probed at characteristic wavelengths at different temperatures, respectively. The wavelength of pump source is $355 \mathrm{~nm}$. 

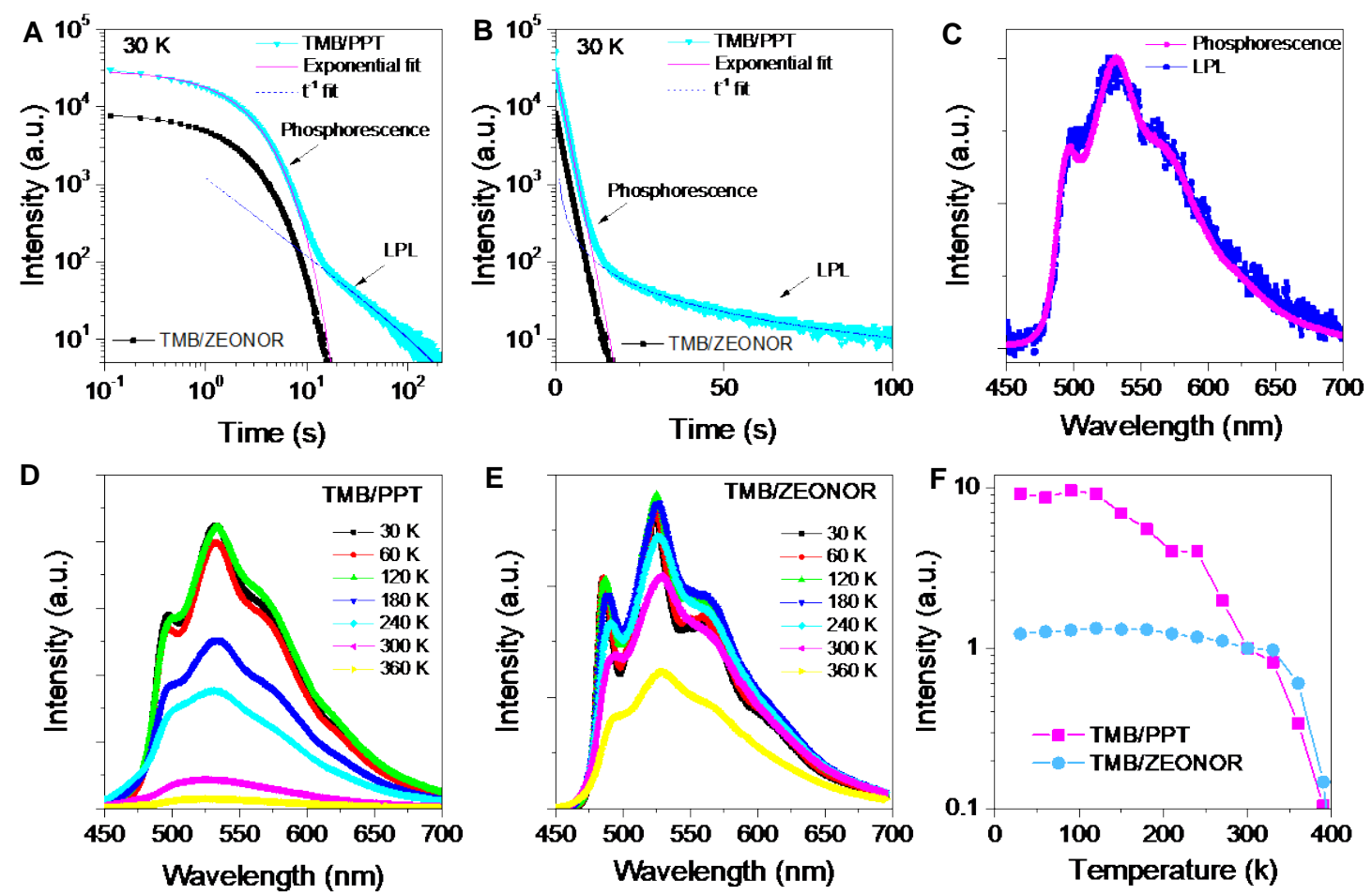

Figure S9. Temperature-dependent PL emission and decay dynamics of TMB/PPT and reference films. (A) Double-logarithmic and (B) single-logarithmic plot of the emission decay profile of the blend films of TMB/PPT and TMB/ZEONOR at $30 \mathrm{~K}$. The decay profile from the TMB/PPT blend film can be reproduced by a phosphorescence emission following exponential decay on a time scale of $<10 \mathrm{~s}$ and a LPL emission following $t^{-1}$ decay at a later stage. (C) Spectra of phosphorescence emission at $1 \mathrm{~s}$ and LPL emission integrated from $20 \mathrm{~s}$ to $100 \mathrm{~s}$. Phosphorescence emission spectra from the blend films of (D) TMB/PPT and (E) TMB/ZEONOR at different temperatures. (F) Temperature dependences of phosphorescence emission intensities from the blend films of (D) TMB/PPT and (E) TMB/ZEONOR, respectively. The data are plotted in the scale normalized to the values at $300 \mathrm{~K}$. 

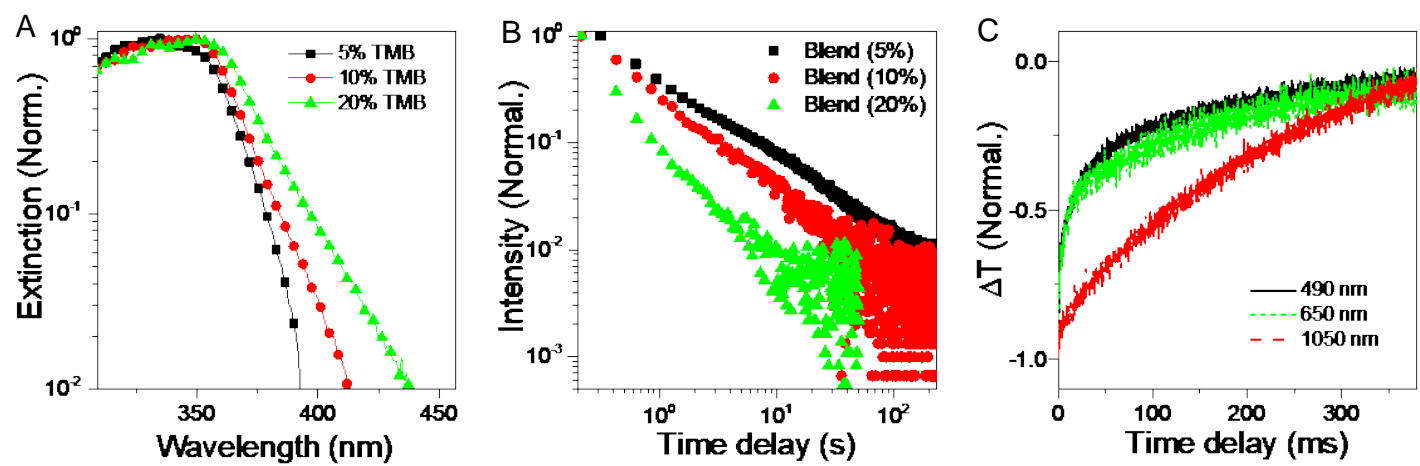

Figure S10. (A) Normalized excitinc spectra of the blend TMB/PPT films with different doping levels of TMB. With incresing the doping level of TMB, the absorption of long-wavelength tail becomes more important due to increased CT transition. The energy of CT transition slightly decreases due to modification of morphology with increasing the doping level of TMB. (B) Normalized kinetics of LPL emission from the blend films of different doping levels of TMB as a reference of Figure $1 \mathrm{C}$ in the main text. (C) Normalized kinetic curves probed at different wavelengths on the ms time scale as a reference of Figure 4B in the main text. 
Table S1. Energy alignment of low-lying excited states with different configurations. Overview of the excitation feature analysis of four types of dimers (intermolecular interaction order: Type I > II > III > IV) generated by MD simulation with one sample for each type. Here ' $D$ ' and 'A' represent that locally-excited states on the site of TMB and PPT, respectively and ' $\mathrm{CT}$ ' means a charge-transfer excited state, and ' 1 ' and ' 3 ' represent singlet and triplet states, respectively. (unit of $\Delta E: \mathrm{eV}$ )

\begin{tabular}{ccccccccc}
\hline \hline \multirow{2}{*}{ state index } & \multicolumn{2}{c}{ Type I } & \multicolumn{2}{c}{ Type II } & \multicolumn{2}{c}{ Type III } & \multicolumn{3}{c}{ Type IV } \\
\cline { 2 - 9 } & feature & $\Delta E$ & feature & $\Delta E$ & feature & $\Delta E$ & feature & $\Delta E$ \\
\hline 1 & ${ }^{3} \mathrm{D}$ & 3.30 & ${ }^{3} \mathrm{D}$ & 3.48 & ${ }^{3} \mathrm{D}$ & 3.39 & ${ }^{3} \mathrm{D}$ & 3.50 \\
2 & ${ }^{3} \mathrm{CT}$ & 3.42 & ${ }^{3} \mathrm{~A}$ & 3.68 & ${ }^{3} \mathrm{~A}$ & 3.64 & ${ }^{3} \mathrm{~A}$ & 3.66 \\
3 & ${ }^{1} \mathrm{CT}$ & 3.57 & ${ }^{3} \mathrm{CT}$ & 3.86 & ${ }^{3} \mathrm{D}$ & 3.90 & ${ }^{3} \mathrm{D}$ & 3.98 \\
4 & ${ }^{3} \mathrm{~A}$ & 3.67 & ${ }^{1} \mathrm{CT}$ & 3.95 & ${ }^{3} \mathrm{~A}$ & 3.95 & ${ }^{3} \mathrm{~A}$ & 4.05 \\
5 & - & - & - & - & ${ }^{3} \mathrm{D}$ & 4.09 & ${ }^{3} \mathrm{D}$ & 4.09 \\
6 & - & - & - & - & ${ }^{3} \mathrm{CT}$ & 4.11 & ${ }^{3} \mathrm{~A}$ & 4.14 \\
7 & - & - & - & - & ${ }^{1} \mathrm{CT}$ & 4.11 & ${ }^{3} \mathrm{D}$ & 4.23 \\
\hline \hline
\end{tabular}




\section{References}

(1) Lin, Z. S.; Kabe, R.; Nishimura, N.; Jinnai, K.; Adachi, C. Organic long-persistent luminescence from a flexible and transparent doped polymer. Adv. Mater. 2018, 30 (45), 1803713.

(2) Xu, Y.; Chen, Q.; Zhang, C.; Wang, R.; Wu, H.; Zhang, X.; Xing, G.; Yu, W. W.; Wang, X.; Zhang, Y.; Xiao, M. Two-photon-pumped perovskite semiconductor nanocrystal lasers. J. Am. Chem. Soc. 2016, 138 (11), 3761-3768.

(3) Wang, R.; Yao, Y.; Zhang, C.; Zhang, Y.; Bin, H.; Xue, L.; Zhang, Z.-g.; Xie, X.; Ma, H.; Wang, X.; Li, Y.; Xiao, M. Ultrafast hole transfer mediated by polaron pairs in all-polymer photovaltaic blends. Nat. Commun. 2019, 10, 398.

(4) Phillips, J. C.; Braun, R.; Wang, W.; Gumbart, J.; Tajkhorshid, E.; Villa, E.; Chipot, C.; Skeel, R. D.; Kale, L.; Schulten, K. Scalable molecular dynamics with NAMD. J. Comput. Chem. 2005, 26 (16), 1781-1802.

(5) Dodda, L. S.; de Vaca, I. C.; Tirado-Rives, J.; Jorgensen, W. L. LigParGen web server: an automatic OPLS-AA parameter generator for organic ligands. Nucleic Acids Research 2017, 45 (W1), W331-W336.

(6) Martinez, L.; Andrade, R.; Birgin, E. G.; Martinez, J. M. PACKMOL: A Package for Building Initial Configurations for Molecular Dynamics Simulations. J. Comput. Chem. 2009, 30 (13), 2157-2164.

(7) Jorgensen, W. L.; Tirado-Rives, J. Potential energy functions for atomic-level simulations of water and organic and biomolecular systems. Proc. Natl. Acad. Sci. U.S.A. 2005, 102 (19), 6665-6670.

(8) Dodda, L. S.; Vilseck, J. Z.; Tirado-Rives, J.; Jorgensen, W. L. 1.14*CM1A-LBCC: Localized Bond-Charge Corrected CM1A Charges for Condensed-Phase Simulations.

J. Phys. Chem. B 2017, 121 (15), 3864-3870.

(9) Frisch, M. J.; G.W., T.; Schlegel, H. B.; Scuseria, G. E.; Robb, M. A.; Cheeseman, J. R.; Scalmani, G.; Barone, V.; Mennucci, B.; Petersson, G. A.; Nakatsuji, H.; Caricato, M.; Li, X.; Hratchian, H. P.; Izmaylov, A. F.; Bloino, J.; Zheng, G.; Sonnenberg, J. L.; Hada, M.; Ehara, M.; Toyota, K.; Fukuda, R.; Hasegawa, J.; Ishida, M.; Nakajima, T.; Honda, Y.; Kitao, O.; Nakai, H.; Vreven, T.; Montgomery, J., J. A. ; Peralta, J. E.; Ogliaro, F.; Bearpark, M.; Heyd, J. J.; Brothers, E.; Kudin, K. N.; Staroverov, V. N.; Keith, T.; Kobayashi, R.; Normand, J.; Raghavachari, K.; Rendell, A.; Burant, J. C.; Iyengar, S. S.; Tomasi, J.; Cossi, M.; Rega, N.; Millam, J. M.; Klene, M.; Knox, J. E.; Cross, J. B.; Bakken, V.; Adamo, C.; Jaramillo, J.; Gomperts, R.; Stratmann, R. E.; Yazyev, O.; Austin, A. J.; Cammi, R.; Pomelli, C.; Ochterski, J. W.; Martin, R. L.; Morokuma, K.; Zakrzewski, V. G.; Voth, G. A.; Salvador, P.; Dannenberg, J. J.; Dapprich, S.; Daniels, A. D.; Farkas, O.; Foresman, J. B.; Ortiz, J. V.; Cioslowski, J.; Fox, D. J. Gaussian 09 Revision D.01. Gaussian Inc. Wallingford CT 2013.

(10) Martin, R. L. Natural transition orbitals. J. Chem. Phys. 2003, 118 (11), 4775-4777. 